\title{
Corrigendum
}

\section{The tumor suppressor neurofibromin confers sensitivity to apoptosis by Ras-dependent and Ras- independent pathways}

\author{
S Shapira, B Barkan, E Friedman, Y Kloog and R Stein \\ Cell Death and Differentiation (2007) 14, 1070. doi:10.1038/sj.cdd.4402124; published online 2 March 2007
}

Correction to: Cell Death and Differentiation advance online publication, 10 November 2006; doi:10.1038/sj.cdd.4402057
The author name E Friedman was published incorrectly in the above-referenced paper. The correct author list is shown above. 